\title{
Prognostic value of nasopharynx tumour volume in local-regional advanced nasopharyngeal carcinoma
}

\author{
Weiqiong Ni, Weixiang Qi, Fei Xu, Weiguo Cao, Cheng Xu, Jiayi Chen, Yunsheng Gao \\ Department of Radiation Oncology, Ruijin Hospital, Shanghai Jiao Tong University, School of Medicine, Shanghai 200025, China \\ Contributions: (I) Conception and design: W Ni, Y Gao, W Qi; (II) Administrative support: W Cao, C Xu, J Chen; (III) Provision of study materials \\ or patients: W Ni, F Xu, Y Gao; (IV) Collection and assembly of data: W Ni, F Xu; (V) Data analysis and interpretation: W Ni, W Qi, Y Gao; (VI) \\ Manuscript writing: All authors; (VII) Final approval of manuscript: All authors. \\ Correspondence to: Yunsheng Gao, MD. Department of Radiation Oncology, Ruijin Hospital, Shanghai Jiao Tong University School of Medicine, 197 \\ Ruijin Second Road, Shanghai 200025, China. Email: gys11856@rjh.com.cn.
}

\begin{abstract}
Background: To investigate the prognostic value of pretreatment primary gross tumor with (GTVp) and without retropharyngeal lymph nodes (GTVnx) for predicting survival outcomes in patients with localregional advanced nasopharyngeal carcinoma (NPC) after intensity-modulated radiation therapy (IMRT).

Methods: From Jan 2012 to Dec 2017, 148 patients with local-regional advanced NPC who had undergone definitive radiotherapy were identified. GTVnx volume and retropharyngeal lymph nodes (GTVrLNs) volume were measured based on registration of MRI with contrast-enhanced CT images. The KaplanMeier method was used for survival analysis. Univariate and multivariate prognostic analyses was performed by using the Cox proportional hazard model. Receiver operating characteristic (ROC) curves were used to identify the cut-off point and assess the prognostic value for GTVnx, GTVp and GTVrLNs.

Results: The median follow-up time for the entire group was 27 months (ranging 7 to 80 months). The 3 -year overall survival (OS) rate was $85 \%$, and the 3 -year local failure-free rate (LFFR), distant failurefree rate (DFFR) and disease-free survival (DFS) rates were 93\%, 81\%, and 73\%, respectively. A positive correlation between GTVnx or GTVp volume and T stage was observed (both $\mathrm{P}<0.001$ ). The 3-year LFFR, OS, and DFS rate, but not for DMFS, in NPC patients with GTVnx $\leq 42.7 \mathrm{~cm}^{3}$ was significantly better than those with $>42.7 \mathrm{~cm}^{3}$ (all $\mathrm{P}<0.05$ ). Multivariate analysis indicated that $\mathrm{GTVnx}$ volume $(\mathrm{P}=0.041)$ was the only independent prognostic factor for LFFR, while age and AJCC stage were two independent prognostic factors for OS.

Conclusions: The GTVnx is an independent prognostic factor for local control, while the prognostic value of GTVrLNs is limited. Physicians are recommended to distinguish between GTVnx and retropharyngeal lymph nodes (RLN) involvement when assessing the risk for local recurrence in advanced NPC.
\end{abstract}

Keywords: Nasopharyngeal carcinoma (NPC); primary tumor volume; prognostic factor; retropharyngeal lymph node (RLN)

Submitted Nov 16, 2019. Accepted for publication Dec 26, 2019.

doi: 10.21037/atm.2020.01.03

View this article at: http://dx.doi.org/10.21037/atm.2020.01.03

\section{Introduction}

Nasopharyngeal carcinoma (NPC) is a malignant tumor with remarkable incidence varying among the regions. Based on GLOBOCAN estimates, high incidence of NPC occurs in China and other Southeastern Asia, but a low incidence in most regions of the world. It is estimated that there are more than 50,000 newly diagnosed cases in China and 65,000 worldwide each year. Until now, radiation therapy remains the mainstay therapy for NPC (1). In twodimensional radiotherapy (2D-RT) era, the pretreatment primary gross tumor (GTVp) volume, including primary nasopharynx tumor and retropharyngeal lymph nodes 
(RLNs), of NPC was an important prognostic factor for survival, particularly for local-regional control (2-4). However, an accurate assessment of the tumor volume of NPC in 2D-RT era is difficult and unavailable, which needs a calculation from a three-dimensional perspective. Recently, along with improvement in modern techniques, three-dimensional conformal radiotherapy (3D-CRT) and intensity-modulated radiation therapy (IMRT) have been extensively applied for the treatment of NPC, which significantly improves tumor control and long-term survival without excessive toxicity, and GTVp is still considered as a key prognostic factor for NPC in modern era $(1,5-7)$. In addition, several recently published data recommended that pretreatment tumor volume could be incorporated into TNM staging system and directed NPC treatment decision (8-10).

However, the primary nasopharynx tumor and the involved RLNs usually are generally outlined together as gross tumor volume, considering the common phenomena of integration $(7,11)$. As a result, the GTVnx and volume of RLNs (GTVrLNs) are usually considered as primary tumor volume when analysis of the effect of primary tumor volume on prognosis is performed $(5,7)$. Currently, MRI has replaced CT as staging criteria because of its higher sensitivity and superiority in identifying tumor extension. And RLN has been classified as N stage (12-16) and could be delineated at CT and MRI fused imaging accurately $(17,18)$. As a result, it is urgently needed to distinguish GTVnx and RLNs involvement when assessing the prognostic value of these factors in advanced NPC. Indeed, several researches were conducted to investigate the prognostic role of RLN metastasis in NPC patients in the past decades, but the results were controversial. Tham et al. demonstrated that RLN metastasis was an independent prognostic factor for distant metastasis in multivariate analysis ( $\mathrm{P}=0.04)$, while both Chen et al. and $\mathrm{Ma}$ et al. found that RLN metastasis had a tendency to affect the DMFS rates of NPC patients $(13,19,20)$. To our best knowledge, the prognostic value of pretreatment primary gross tumor without retropharyngeal lymph nodes (GTVnx) for predicting survival outcomes in patients with local-regional advanced NPC remains undetermined. Therefore, the aim of current report is mainly to address the prognostic value of pretreatment volume of GTVp, GTVnx, and GTVrLNs for predicting survival outcomes in local-regional NPC patients after IMRT according to an institutional protocol. We also analyze the prognosis of summation of primary GTVnx and GTVrLNs in order to find a cut off value above which the impact on survival parameters was statistically significant.

\section{Methods}

\section{Study design and eligibility}

The inclusion criteria for this retrospective study were: (I) newly-onset, biopsy-proven and non-metastatic NPC, (II) uniform protocol of definitive radiotherapy and/or combined chemotherapy, (III) available pre-treatment MRI, (IV) and discrimination of RLNs from primary tumor. From Jan. 2012 to Dec. 2017, a total of 148 eligible patients were enrolled in this study. Table 1 listed the baseline characteristics of included patients. The patients included $116(78.4 \%)$ males and 32 (21.6\%) females. The median age was 53 years (13-84 years). This study was approved by the Institutional Review Board of the Ruijin Hospital affiliated to Shanghai Jiao Tong University School of Medicine, China. Individual written informed consent was not required because of the retrospective nature of the study.

\section{Treatment planning and delivery}

All patients were immobilized in the supine position with a tailored head-shoulder thermoplastic mask, followed by a CT simulation. CT simulation (Brilliance Big Bore, Phillips, Amsterdam, Netherlands) was performed at a slice thickness of $3 \mathrm{~mm}$ from the head to $5 \mathrm{~cm}$ below the sternoclavicular joint. The target volumes were delineated slice-by-slice using an individualized protocol that complied with the International Commission on Radiation Units and Measurements reports 50 and 62 as previously described $(11,21-23)$.

The primary tumor and the involved RLNs on each axial CT slice were both delineated on the planning system according to the pretreatment contrast axial T1-weighted MRI and CT fused images. In this study, the volume of RLNs was separated from primary nasopharynx tumor (Figure 1). For patients given induction chemotherapy, the targets of primary tumor volume were contoured based on the pretreatment MRI images. The inverse IMRT planning system developed by Philips (Madison, WI, USA), the Pinnacle version 8.0 planning system was used to do all treatment plans. The GTVnx, volume of RLNs (in cubic centimeters) was calculated subsequently by the same software. The primary gross tumor volume (GTVp) was obtained by summing the GTVnx and volume of RLNs. 
Table 1 Characteristics of 148 patients

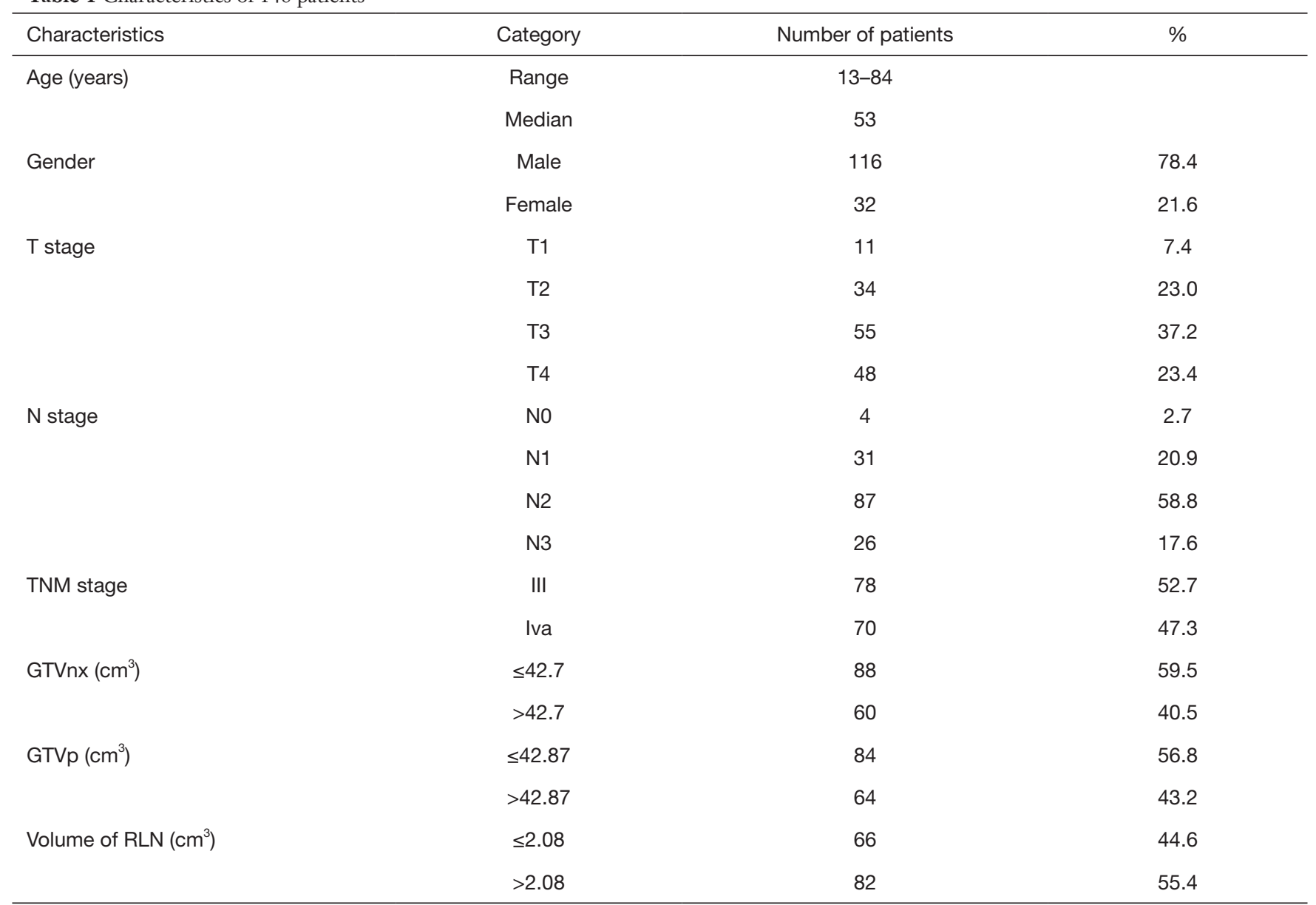

\section{Radiotherapy}

All patients were treated using IMRT. The planning target volume of the primary nasopharynx tumor volume including retropharyngeal lymph nodes (PTV-G) and positive lymph nodes (PTV-LN) received 66-70.4 Gy and 70.4 Gy in 30-32 fractions, whereas 60 and 54 Gy in 30-32 fractions were prescribed to the PTVs of CTV1 and CTV2, or received 70 Gy for PTV-G at 2.12 Gy/fraction, 66 Gy for PTV-LN at 2 Gy/fraction whereas 60 and 54 Gy for PTVs of CTV1 and CTV2 delivered in 33 fractions, or received 70 Gy for PTV-G at 2 Gy/fraction, 66 Gy for PTV-LN and 60 and 54 Gy for PTVs of CTV1 and CTV2 delivered in 35 fractions. RT was delivered over one fraction daily, 5 days per week.

\section{Chemotherapy regimen}

Based on the treatment guidelines for NPC at our hospital, concurrent chemotherapy +/- induction chemotherapy or adjuvant chemotherapy was part of the treatment plan for all patients, except those who were unwilling to receive chemotherapy due to poor perform status for local-regional advanced NPC. Induction chemotherapy was consisted of paclitaxel $\left(135 \mathrm{mg} / \mathrm{m}^{2}\right)$ or docetaxel $\left(75 \mathrm{mg} / \mathrm{m}^{2}\right)$ and cisplatin $\left(75 \mathrm{mg} / \mathrm{m}^{2}\right)$ every 3 weeks for $2-3$ cycles. Concurrent chemotherapy was consisted of cisplatin administered every 3 weeks $\left(100 \mathrm{mg} / \mathrm{m}^{2}\right)$ or weekly $\left(40 \mathrm{mg} / \mathrm{m}^{2}\right)$ during RT.

\section{followed-up and statistical analysis}

After RT, all patients were assessed every 3 months during the first 2 years, and every 6 months thereafter until death. All events were measured from the date of completion of radiation therapy until documented treatment failure or the last follow-up visit. The primary end-point was: local failure-free rate (LFFR-persistence/recurrence at nasopharynx). The second end-points were overall survival 

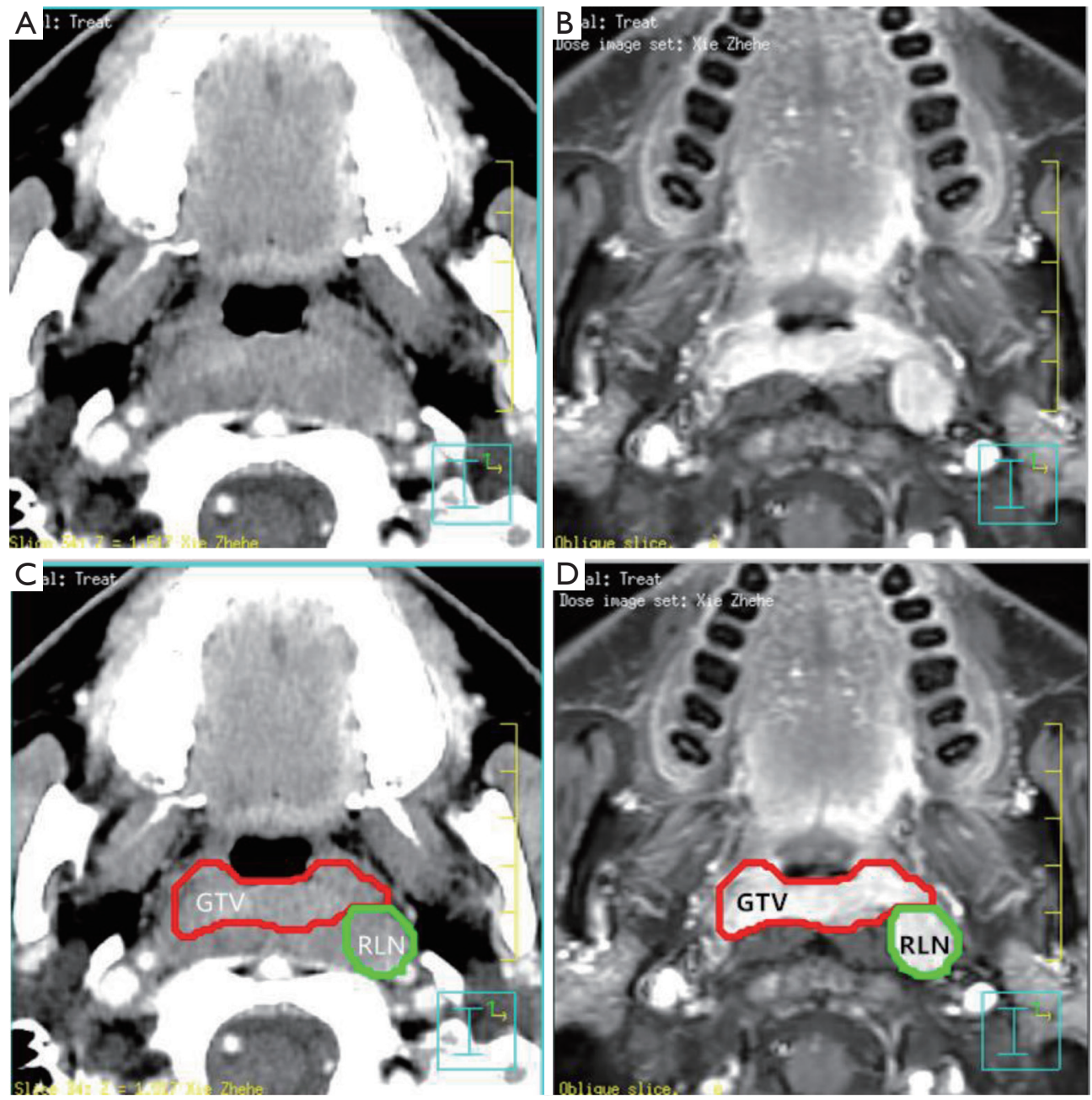

Figure 1 The volume of RLNs (in green), which was separated from primary nasopharynx tumor (in red), was both delineated on the planning system according to the pretreatment contrast axial T1-weighted MRI (B,D) and CT fused images (A,C). RLN, retropharyngeal lymph node.

(OS-death due to any cause), disease-free survival (DFSstaying free of disease after radiotherapy), and distant failure-free rate (DFFR-disease metastasis at distant sites).

The Kruskal-Wallis test was used to examine the differences in GTVp/GTVnx between T stages. Actuarial rates of LFFR, OS, DFS and DFFR rates were calculated with the Kaplan-Meier method, and compared by using log-rank test. Receiver operating characteristic (ROC) curves were used to identify the cut-off values for above endpoints. The areas under the ROC curve (AUC) were used to assess the prognostic value of GTVnx, GTVp. To identify potential factors associated with LFFR, OS, PFS and DFFR. Univariate analyses and multivariate logistic regression model were used to identify independent risk factors. A forward stepwise approach was used to enter new terms into the model, with a limit of $\mathrm{P}<0.05$ to enter the terms and the most significant term entered first. All statistical tests were two-sided and $\mathrm{P}$ values of 0.05 or less were considered significant. Analyses were performed by use of the NCSS and MedCalc statistical software.

\section{Results}

\section{Treatment outcomes}

The median follow-up time for the entire group was 27 months (range 7 to 80 months). At the time of this analysis, a total of 4 cases developed regional recurrence, 9 
Table 2 T stage and GTVp, volume of RLNs and GTVnx (mean \pm SD $\left.\mathrm{cm}^{3}\right)$

\begin{tabular}{lcccc}
\hline Variables & T1 & T2 & T3 & T4 \\
\hline GTVnx & $15.687 \pm 8.281$ & $18.717 \pm 14.022$ & $35.147 \pm 22.459$ & $85.625 \pm 40.484$ \\
Volume of RLNs & $1.008 \pm 1.862$ & $3.886 \pm 4.286$ & $3.309 \pm 4.715$ & $3.711 \pm 5.100$ \\
GTVp & $16.696 \pm 8.212$ & $22.604 \pm 15.003$ & $38.456 \pm 23.625$ & $89.336 \pm 42.187$ \\
\hline
\end{tabular}

$R L N$, retropharyngeal lymph node.

patients experienced local failure. In addition, 24 patients developed distant metastasis. There were a total of 17 deaths: 9 patients died from distant metastasis, 6 patients died from progression of local disease after recurrence, and the causes of death for the additional 2 cases were unknown. The 3-year OS rate of whole cohort was $85 \%$, and the 3 -year LFFR, DFFR, and DFS rates were $93 \%, 81 \%$, and $73 \%$, respectively.

\section{Distribution of tumor volume by T stage}

The mean GTVnx of the whole series was $46.30 \mathrm{~cm}^{3}$ (range, $\left.1.67-245.79 \mathrm{~cm}^{3}\right)$. Table 2 showed that the distribution of GTVp volume, GTVrLNs volume and GTVp volume according to $\mathrm{T}$ stage. Although the variation within the same T stage was wide, the mean GTVnx and GTVp volume increased in consistent with advanced $\mathrm{T}$ stage: T1 $\left(\mathrm{n}=11,15.687 \mathrm{~cm}^{3}\right) ; \mathrm{T} 2\left(\mathrm{n}=34,18.717 \mathrm{~cm}^{3}\right) ;$ T3 $(\mathrm{n}=55$, $\left.35.147 \mathrm{~cm}^{3}\right)$; and T4 $\left(\mathrm{n}=48,85.625 \mathrm{~cm}^{3}\right)$. The mean GTVp in different T stage: T1 $\left(16.696 \mathrm{~cm}^{3}\right)$; T2 $\left(22.604 \mathrm{~cm}^{3}\right)$; T3 $\left(38.456 \mathrm{~cm}^{3}\right)$; and T4 $\left(89.336 \mathrm{~cm}^{3}\right)$. Primary tumor classified as a more advanced $\mathrm{T}$ stage had a significantly larger GTVp, GTVnx than those with an early T stage $(\mathrm{P}<0.001)$. There was no significant correlation between the volume of RLNs and the T stage.

\section{Identification of $G T V p / G T V n x$ cut-off value}

ROC curve was used to search for the best cut-off value of the GTVnx, GTVp on for OS prediction. According to ROC curve results, patients were stratified into two different groups for further analyses. For GTVnx, 3-year LFFR in patients with GTVnx $\leq 42.7$ and $>42.7 \mathrm{~cm}^{3}$ were $97 \%$ and $89 \%(\mathrm{P}=0.026)$, OS, DFFR and DFS were $94 \%$ and $69 \%(\mathrm{P}=0.007), 83 \%$ and $76 \%(\mathrm{P}=0.59)$, and $79 \%$ and $61 \%$, respectively ( $\mathrm{P}=0.04$, Figure 2$)$. For GTVp, 3-year LFFR in patients with PTV $\leq 42.87$ and $>42.87 \mathrm{~cm}^{3}$ were $97 \%$ and $89 \%(\mathrm{P}=0.034)$, OS, DFFR and DFS were $94 \%$ and $70 \%(\mathrm{P}=0.01), 84 \%$ and $75 \%(\mathrm{P}=0.42)$, and were $80 \%$ and $60 \%$, respectively $(\mathrm{P}=0.026)$.

\section{Pairwise comparison of ROC curves among T stage, GTVP, GTVnx for LFFR, OS, DFFR and DFS}

Pairwise comparison of ROC curves among $\mathrm{T}$ stage, GTVp, GTVnx for LFFR, OS, DFFR, DFS showed GTVnx has a better predictive effect than GTVp in overall survival $(\mathrm{P}=0.0020)$. There was no significant difference in the predictive effect of T stage and GTVnx on LFFR, DFFR and DFS. In addition, the predictive effects of $T$ stage, GTVnx, GTVp for LFRR, DFFR, and DFS were all insignificantly different (Tables 3,4).

\section{Prognostic factors}

The value of various potential prognostic factors, including gender, age, T- and N-classification, TNM stage, GTVnx, GTVp, volume of RLNs (GTVrLNs), on predicting LFFR, DFFR, DFS and OS were evaluated. Univariate analysis demonstrated that age, GTVnx, GTVp were significant prognostic factors for predicting LFFR; age, GTVnx, GTVp, TNM stage for OS; TNM stage for DFFR; and TNM, age, GTVp and GTVnx for DFS (Table 5). Multivariate analysis showed GTVnx was the only independent prognostic factor for local control $(\mathrm{HR}=5.24$, 95\% CI, 1.074-25.57, $\mathrm{P}=0.041)$, age $(\mathrm{HR}=4.98,95 \% \mathrm{CI}$, $1.81-13.67, \mathrm{P}=0.0019)$ and $\mathrm{TNM}$ stage $(\mathrm{HR}=4.66,95 \% \mathrm{CI}$, 1.06-20.41, $\mathrm{P}=0.041$ ) were significant prognostic factors for predicting overall survival (Table 5).

\section{Discussion}

Currently, the treatment decision and prognostic prediction for NPC is mainly dependent on clinical stage. Although tumor volume has not been included in the clinical staging of NPC, multiple researches have demonstrated that volume of GTV-primary is significantly associated with survival and local recurrence in NPC after radiotherapy. 
A

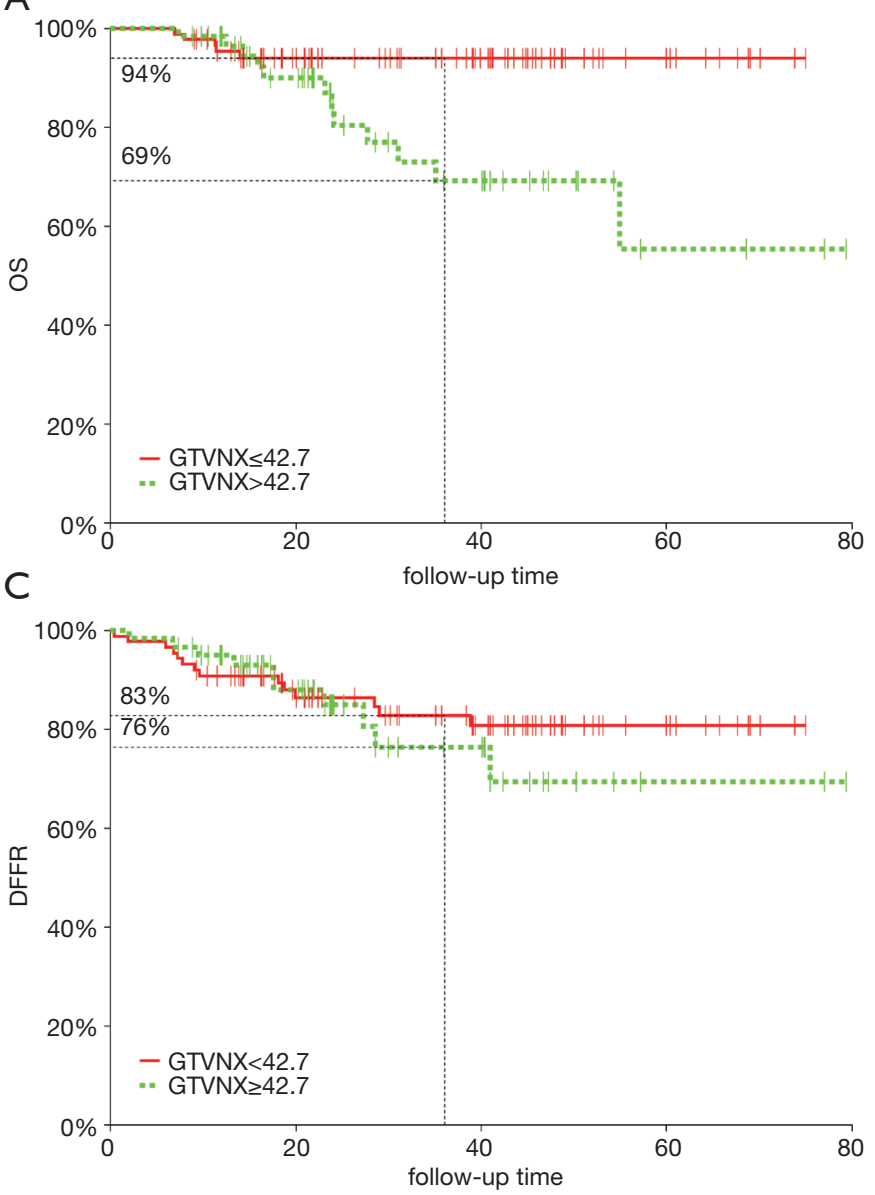

B
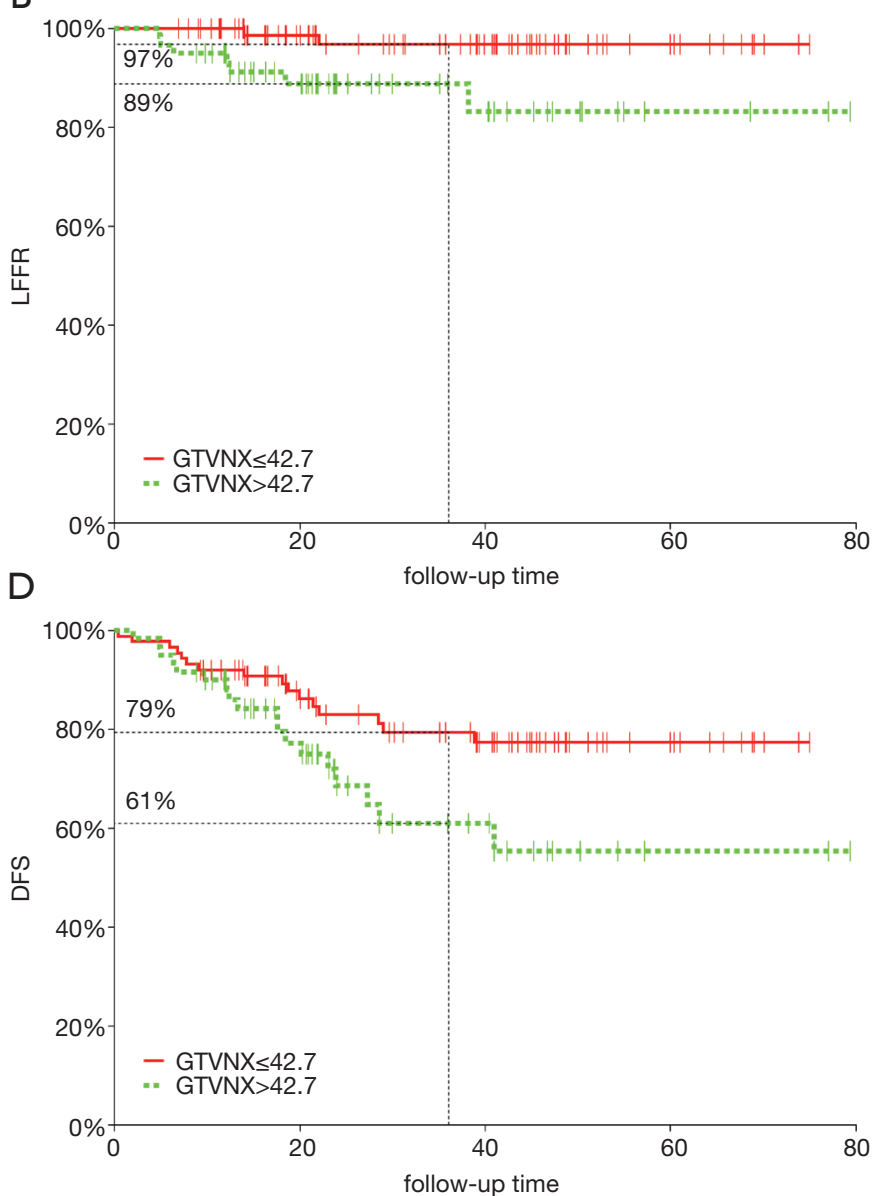

Figure 2 Three-year of survival curve of (A) overall survival (OS), (B) local failure-free rate (LFFR), (C) distant failure-free rate (DFFR), and (D) disease free survival (DFS) for GTVnx $\leq 42.7$ and $>42.7 \mathrm{~cm}^{3}$.

Table 3 Summary of AUC of T stage, GTVnx, GTVp for LFFR, OS, DFFR and DFS

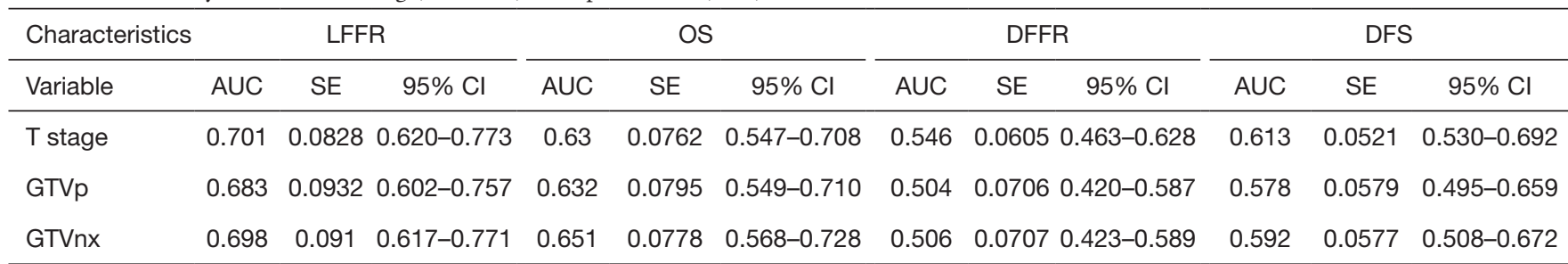

AUC, areas under the ROC curve; LFFR, local failure-free rate; OS, overall survival; DFFR, distant failure-free rate; DFS, disease-free survival.

Sze et al. (5) found that with every $1 \mathrm{~cm}^{3}$ increase in tumor volume, local failure increased by $1 \%$. In a retrospective study conducted by Wu et al. the authors found that GTVprimary volume was an independent prognostic factor for local control, distant metastasis, DFS, and overall survival in NPC (24). Similar results were also observed in a subsequent study performed by Qin et al. (25). However, to our best knowledge, most of these included trials define the volume of GTV-primary as the combined volume of GTVnx and RLNs, while several published research show 
Table 4 Pairwise comparison of ROC curves among T stage, GTVp, GTVnx for LFFR, OS, DFFR, DFS

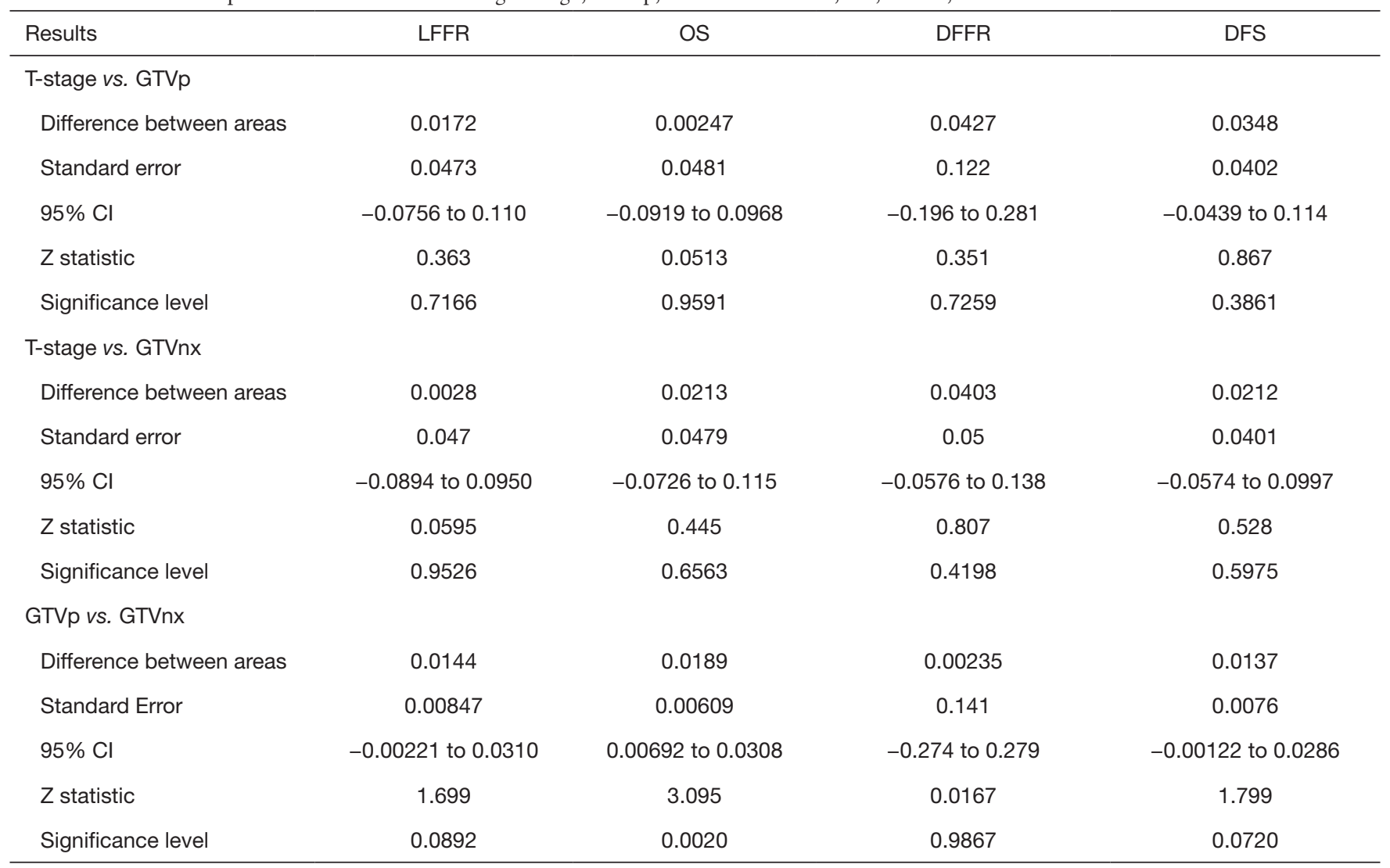

ROC, receiver operating characteristic; LFFR, local failure-free rate; OS, overall survival; DFFR, distant failure-free rate; DFS, disease-free survival.

that RLN metastasis is a risk factor for distant metastasis in NPC, but not for local regional recurrence (12-14). As a result, regarding the volume of RLNs as primary gross tumor when evaluating the prognostic effects of primary gross tumor volume in NPC would be inappropriate. Therefore, we aim to explore the prognostic value of pretreatment nasopharynx tumor volume (GTVnx) with or without RLNs for predicting survival outcomes in patients with local-regional NPC after definitive IMRT.

In consistent with previous results, univariate analysis of the present study shows that both volume of GTVp and GTVnx are significantly prognostic factors for LFFR, OS and PFS, but not for DFFR. However, in multivariate analysis, only GTVnx, but not for GTVp, is a significant prognosticator for local control $(\mathrm{HR}=5.24,95 \% \mathrm{CI}$, 1.074-25.57, $\mathrm{P}=0.041)$. As for the prognostic role of GTVrLNs, no significant association is found between GTVrLNs volume and LFFR, OS, DFS, or DFFR. Therefore, we think that the volume of RLNs should not be regarded as primary gross tumor when evaluating the prognostic effects of primary gross tumor volume in NPC, although multiple studies also found that volume of GTVprimary (including both GTVnx and RLNs) could also be a risk factor for survival and recurrence. One possible explanation for this finding is that the volume of RLNs is much smaller than the volume of the nasopharynx tumor, and dosimetric advantage of application of IMRT and usage of chemoradiotherapy significantly increases the local-regional control rate for local advanced NPC, which might make no significant differences between GTVnx and GTVp.

Though best cut-off value of the GTVp, GTVp was obtained for survival analysis, they had identical predictive effects on prognosis as comparing to $\mathrm{T}$ staging. However, tumor volume overlaps between tumors at different $\mathrm{T}$ categories, especially T3/T4 tumors, their volumes vary widely. And tumor volume heterogeneity of the same $\mathrm{T}$ classification could potentially cause much difficulty for predicting prognosis. Although the available data showed 


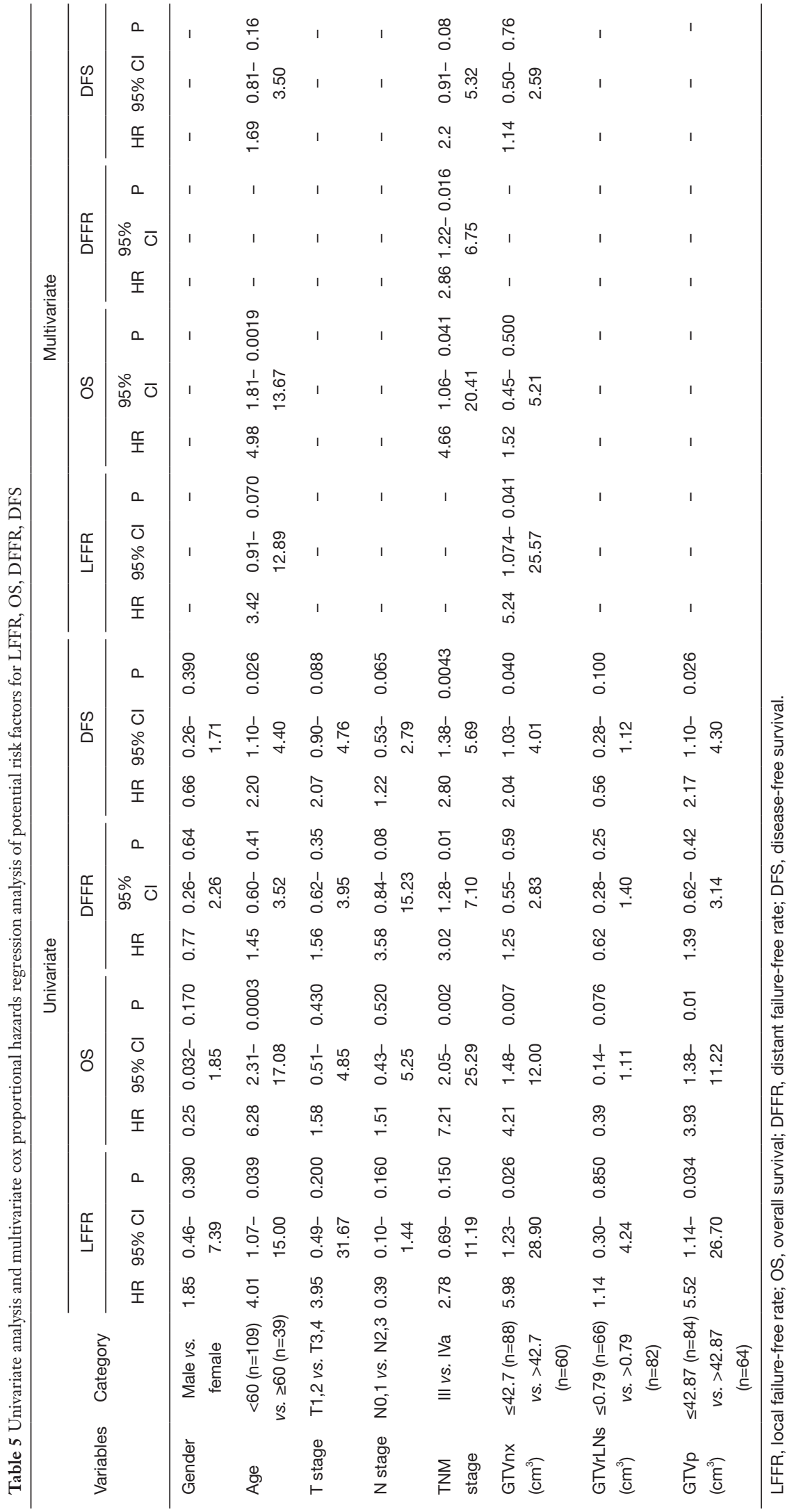


GTVp and T staging were closely related and GTVp could be incorporated into the TNM system by which the treatment outcomes would be more accurately and predicted (12-16), the cut-off value of tumor volume that predicts a poor prognosis has been reported inconsistently, which might be one main reason for not incorporating tumor volume into the clinical staging of NPC yet. Feng et al. (1) reported that primary tumor volume (including both primary nasopharyngeal tumor and retropharyngeal nodes) $>40 \mathrm{~mL}$ was an adverse prognostic factor for the 5 -year local regional control (LRC) rate (RR 2.454, $\mathrm{P}=0.002)$. In a retrospective study conducted by $\mathrm{Wu}$ et al. (24) showed that primary gross tumor volume (including both primary nasopharyngeal tumor and retropharyngeal nodes) $>49 \mathrm{~mL}$ is an independent prognostic factor for local control based on 321 NPC patients. Our data showed that a volume of $42.7 \mathrm{~cm}^{3}$ was a useful cut-off point to categorize patients into different prognostic groups. NPC patients with GTVnx $\leq 42.7 \mathrm{~cm}^{3}$ had better 3-year LFFR, OS, and DFS $(\mathrm{HR}=5.98,95 \% \mathrm{CI}, 1.23-28.90, \mathrm{P}=0.026$; $\mathrm{HR}=4.21$, 95\% CI, 1.48-12.00, $\mathrm{P}=0.007$ and $\mathrm{HR}=2.04,95 \% \mathrm{CI}$, $1.03-4.01, \mathrm{P}=0.04$ respectively). The cut-off volume in the present study is smaller than reported by Feng $M$. et al, the main reason for this difference is that all of the included NPC is locally advanced (Stage III or IV), while nearly 30\% of included patients in the Feng' study is early stage I or II. Based on our findings, for patients with GTVnx $>42.7 \mathrm{~cm}^{3}$, altered fractionation or dose escalating or boosting tumors with pretreatment volume could be recommended to further improve outcomes and local-regional control of NPC patients (26-28).

The previous methods of tumor delineation and volume calculation were not very accurate and varied, which lead to inconsistent research results (1-5,7-10). Although TPS could obtain a more accurate tumor treatment volume, induction chemotherapy was often selected as first line treatment scheme (29-31), and CT simulation images usually were collected after chemotherapy, it was more difficult to obtain the accurate pretreatment tumor volume because it was obviously reduced after induction chemotherapy. In our study, for local-regional advanced NPC, the volume exceeds $42.7 \mathrm{~cm}^{3}$ predicting the unfavorable prognosis, and this part of the patient accounts for about $40 \%$. The staging system should be suitable for all patients. If GTVnx was considered in the staging system, it couldn't direct patient's treatment scheme selection, which was one of key roles of staging system. Pairwise comparison of ROC curves among T stage, GTVp, GTVnx for LFFR, OS, DFFR, PFS, this research showed GTVnx has a better predictive effect than GTVp in overall survival, $\mathrm{P}=0.0020$, which might be associated with volumes of RLNs, which adversely affects the prognosis effect of the GTVnx. The volume of RLNs should be excluded when the effects of GTVnx on the prognosis were evaluated. There was no significant difference in the predictive effect of T stage and GTVp on LFFR, DFFR and DFS. In addition, the predictive effects of T stage, GTVnx, GTVp for LFRR, DFFR, and DFS were all insignificantly different. All in a word, it was not necessary to incorporated GTV volume into tumor staging under the currently available diagnostic and therapeutic methods.

The major patterns of treatment failure in this group are still the distant metastasis, followed by the local regional failure. Based on the available clinical data, induction chemotherapy followed by concurrent chemoradiotherapy is recommended as the first line treatment option for localregional advanced NPC patients in order to improve the survival and local control (28-30). However, pretreatment GTVp and GTVnx often shrink significantly after induction chemotherapy and concurrent chemoradiotherapy in NPC patients. Until now, no published data is available regarding the prognostic effect of post-treatment GTVp and GTVnx after chemoradiotherapy in NPC, and further studies are still needed to clearly investigate this issue.

There were several limitations that must be addressed in the current study. First of all, the treatment regimens of included patients are varied, although most local advanced patients received induction chemotherapy and concurrent chemoradiotherapy, which might increase the heterogeneity of the included patients. Another limitation is that this is a retrospective study, thus the inherent bias could not be excluded. In addition, the sample of the present study is relatedly small and follow-up time (median 27 months) is relatively short.

\section{Conclusions}

In conclusion, in this retrospective study, we found that GTVnx was a significant prognostic factor for local control while the prognostic value of RLNs volume was limited. Physicians were recommended to distinguish between GTVnx and RLN involvement when assessing the risk for local recurrence in advanced NPC. Further investigations were warranted to confirm our findings.

\section{Acknowledgments}

Funding: None. 


\section{Footnote}

Conflicts of Interest: The authors have no conflicts of interest to declare.

Ethical Statement: The authors are accountable for all aspects of the work in ensuring that questions related to the accuracy or integrity of any part of the work are appropriately investigated and resolved. This study was approved by the Institutional Review Board of the Ruijin Hospital affiliated to Shanghai Jiao Tong University School of Medicine, China. Individual written informed consent was not required because of the retrospective nature of the study.

Open Access Statement: This is an Open Access article distributed in accordance with the Creative Commons Attribution-NonCommercial-NoDerivs 4.0 International License (CC BY-NC-ND 4.0), which permits the noncommercial replication and distribution of the article with the strict proviso that no changes or edits are made and the original work is properly cited (including links to both the formal publication through the relevant DOI and the license). See: https://creativecommons.org/licenses/by-nc-nd/4.0/.

\section{References}

1. Feng $M$, Wang W, Fan Z, et al. Tumor volume is an independent prognostic indicator of local control in nasopharyngeal carcinoma patients treated with intensitymodulated radiotherapy. Radiat Oncol 2013;8:208.

2. Chua DT, Sham JS, Kwong DL, et al. Volumetric analysis of tumor extent in nasopharyngeal carcinoma and correlation with treatment outcome. Int J Radiat Oncol Biol Phys 1997;39:711-9.

3. Shen C, Lu JJ, Gu Y, et al. Prognostic Impact of Primary Tumor Volume in Patients With Nasopharyngeal Carcinoma Treated by Definitive Radiation Therapy. The Laryngoscope 2008;118:1206-10.

4. Yao X, Gong G, Zuo G, et al. Study of the dose-volume parameters variation in tumor target volumes and organs at risk during nasopharyngeal carcinoma radiotherapy applying deformation registration. Transl Cancer Res 2019;8:2886-92.

5. Sze WM, Lee A WM, Yau TK, et al. Primary tumor volume of nasopharyngeal carcinoma: prognostic significance for local control. Int J Radiat Oncol Biol Phys 2004;59:21-7.
6. Li JX, Huang SM, Jiang XH, et al. Local failure patterns for patients with nasopharyngeal carcinoma after intensitymodulated radiotherapy. Radiat Oncol 2014;9:87.

7. Guo R, Sun Y, Yu XL, et al. Is primary tumor volume still a prognostic factor in intensity modulated radiation therapy for nasopharyngeal carcinoma? Radiother Oncol 2012;104:294-9.

8. Chen MK, Chen HH, Liu JP, et al. Better prediction of prognosis for patients with nasopharyngeal carcinoma using primary tumor volume. Cancer 2004;100:2160-6.

9. Chen C, Fei Z, Pan J, et al. Significance of Primary Tumor Volume and T-stage on Prognosis in Nasopharyngeal Carcinoma Treated with Intensity-modulated Radiation Therapy. Jpn J Clin Oncol 2011;41:537-42.

10. Lee CC, Chu ST, Ho HC, et al. Primary tumor volume calculation as a predictive factor of prognosis in nasopharyngeal carcinoma. Acta Otolaryngol 2008;128:93-7.

11. Ni W, Gao Y, Xu F, et al. The maximum diameter of cervical lymph node was not a prognostic factor for localregional advanced nasopharyngeal carcinoma treated with intensity modified radiotherapy. Transl Cancer Res 2019;8:802-10.

12. Tang L, Li L, Mao Y, et al. Retropharyngeal lymph node metastasis in nasopharyngeal carcinoma detected by magnetic resonance imaging: Prognostic value and staging categories. Cancer 2008;113:347-54.

13. Ma J, Liu L, Tang L, et al. Retropharyngeal Lymph Node Metastasis in Nasopharyngeal Carcinoma: Prognostic Value and Staging Categories. Clin Cancer Res 2007;13:1445-52.

14. Ma SX, Zhou T, Huang Y, et al. The efficacy of first-line chemotherapy in recurrent or metastatic nasopharyngeal carcinoma: a systematic review and meta-analysis. Ann Transl Med 2018;6:201.

15. Ng WT, Lee AWM, Kan WK, et al. N-staging by magnetic resonance imaging for patients with nasopharyngeal carcinoma: Pattern of nodal involvement by radiological levels. Radiother Oncol 2007;82:70-5.

16. Amin MB, Greene FL, Edge SB, et al. The Eighth Edition AJCC Cancer Staging Manual: Continuing to build a bridge from a population-based to a more "personalized" approach to cancer staging. CA Cancer J Clin 2017;67:93-9.

17. Wang XS, Hu CS, Ying HM, et al. Patterns of Retropharyngeal Node Metastasis in Nasopharyngeal Carcinoma. Int J Radiat Oncol Biol Phys 2009;73:194-201.

18. Wang X, Li LG, Hu CS, et al. Patterns of level II node 
metastasis in nasopharyngeal carcinoma. Radiother Oncol 2008;89:28-32.

19. Chen KW, Wang WY, Liang WM, et al. The volume of retropharyngeal nodes predicts distant metastasis in patients with advanced nasopharyngeal carcinoma. Oral Oncol 2011;47:1171-5.

20. Tham IW, Hee SW, Yap SP, et al. Retropharyngeal nodal metastasis related to higher rate of distant metastasis in patients with $\mathrm{N} 0$ and $\mathrm{N} 1$ nasopharyngeal cancer. Head Neck 2009;31:468-74.

21. Jones D. ICRU Report 50-Prescribing, Recording and Reporting Photon Beam Therapy. Medical Physics 1994;21:833-4.

22. Morgan-Fletcher LS. Prescribing, recording and reporting photon beam therapy (supplement to ICRU report 50) (Report 62). Br J Radiol 2001;74:294.

23. Chinese nasopharyngeal carcinoma staging Committee. The consensus 2010 nasopharyngeal carcinoma IMRT target and dose expert design guidelines. Chin J Radiat Oncol 2011;20:267-9.

24. Wu Z, Su Y, Zeng RF, et al. Prognostic value of tumor volume for patients with nasopharyngeal carcinoma treated with concurrent chemotherapy and intensity-modulated radiotherapy. J Cancer Res Clin Oncol 2014;140:69-76.

25. Qin L, Wu F, Lu H, et al. Tumor Volume Predicts Survival Rate of Advanced Nasopharyngeal Carcinoma Treated with Concurrent Chemoradiotherapy. Otolaryngol Head

Cite this article as: $\mathrm{Ni}$ W, Qi W, Xu F, Cao W, Xu C, Chen J, Gao Y. Prognostic value of nasopharynx tumour volume in local-regional advanced nasopharyngeal carcinoma. Ann Transl Med 2020;8(5):223. doi: 10.21037/atm.2020.01.03
Neck Surg 2016;155:598-605.

26. Xu F, Ni W, Gao Y, et al. The dose perfraction as a significant prognostic factor in nasopharyngeal carcinoma (NPC) treated with Intensity Modulated Radiation Therapy (IMRT). Int J Radiat Oncol Biol Phys 2018;102:e373-4.

27. Kwong DLW, Sham JST, Leung LHT, et al. Preliminary results of radiation dose escalation for locally advanced nasopharyngeal carcinoma. Int J Radiat Oncol Biol Phys 2006;64:374-81.

28. Wong FCS, Ng AWY, Lee VHF, et al. Whole-Field Simultaneous Integrated-Boost Intensity-Modulated Radiotherapy for Patients With Nasopharyngeal Carcinoma. Int J Radiat Oncol Biol Phys 2010;76:138-45.

29. Ribassin-Majed L, Marguet S, Lee AWM, et al. What Is the Best Treatment of Locally Advanced Nasopharyngeal Carcinoma? An Individual Patient Data Network MetaAnalysis. J Clin Oncol 2017;35:498-505.

30. Sun Y, Li WF, Chen NY, et al. Induction chemotherapy plus concurrent chemoradiotherapy versus concurrent chemoradiotherapy alone in locoregionally advanced nasopharyngeal carcinoma: a phase 3, multicentre, randomised controlled trial. Lancet Oncol 2016;17:1509-20.

31. Zhang Y, Chen L, Hu G, et al. Gemcitabine and cisplatin induction chemotherapy in nasopharyngeal carcinoma. $\mathrm{N}$ Engl J Med 2019;381:1124-35. 